\title{
A PROBLEM IN THE OPTIMAL DESIGN OF NETWORKS UNDER TRANSVERSE LOADING
}

\author{
BY \\ ELIO CABIB AND CESARE DAVINI (Università di Udine, Italy) \\ AND \\ CHONG-QING RU (Peking University, China)
}

Introduction. In this paper the simpler problem in the optimal design of tension structures is considered. Let a square inextensible network undergo given transverse loads and be prestressed in its plane by means of tractions applied along the boundary. We want to study the optimal distribution of tractions in order to minimize the compliance of the net when the total pretraction load and two positive lower and upper bounds to the pretractions are given. Attention is addressed to general issues; we discuss existence and uniqueness and provide a description of certain qualitative properties of the solution.

The compliance of the net is defined as the work done by the loads evaluated in the equilibrium configuration. It is then a functional of the pretractions and these, in their turn, are the coefficients of an equilibrium operator. Thus, the problem reduces to studying the minimization of a cost functional defined over a certain class of operators, as is customary in many other optimization problems [1-12]. The theory of $G$-convergence (see, e.g., $[13,14]$ ) proves to be the natural setting to discuss the existence of solutions for these problems. At this point, however, because of the constancy of the pretractions along the fibers (cf. [15]) $G$-convergence is in the peculiar circumstance of being equivalent to weak convergence, and the topological properties that are required for a solution to exist can be assessed in a more familiar context. The situation is close to that encountered in dealing with the one-dimensional problems where $G$-convergence is equivalent to weak convergence of the inverses of the operator coefficients, $[16,17]$, so that one could in fact obtain existence results without mentioning $G$-convergence. Velte and Villaggio [18], for instance, follow this approach in a paper which raised our interest in the present problem. This distinguished feature of $G$-convergence in one dimension, however, does not carry over to a higher dimension. So, the fact that a property of this sort holds in the present case gives the problem a special location in structural optimization.

After proving the existence of optimal pretractions, we study other features of the solutions. In particular, by making use of Kuhn-Tucker conditions, it is possible to show that all solutions correspond to the same configuration of the net and that the Received March 29, 1989. 
pretractions must reach the minimum value near the edge of the net. Next, conditions sufficient for uniqueness are outlined and cases where nonunique optimal pretractions occur are described. Although these results are not exhaustive, they provide insight into the problem of uniqueness.

1. Setting of the problem and existence of solutions. We consider a network occupying the unit square $\Omega=(0,1) \times(0,1)$ and made of fibers uniformly distributed and oriented along directions parallel to the sides of the square. Since extensibility of the fibers plays no role in the linearized problem that is considered in the sequel, one can assume that they are inextensible, for definiteness. The fibers are prestressed through the application of forces per unit length $\sigma_{1}=\sigma_{2}\left(x_{2}\right)$ and $\sigma_{2}=\sigma_{2}\left(x_{1}\right)$ along the boundary, so that the network is capable of withstanding a given transverse loading $q$.

Let $\sigma$ denote the pair $\left(\sigma_{1}, \sigma_{2}\right)$, and consider the linearized equilibrium problem for a net supported along the edges

$$
\begin{array}{ll}
\sigma_{1} w,_{11}+\sigma_{2} w,_{22}=-q, & \text { in } \Omega, \\
w=0, & \text { on } \partial \Omega,
\end{array}
$$

where $w$ is the transverse displacement and the load $q$ is taken in $L^{2}(\Omega)$, with $\|q\| \neq 0$. Here, it is of interest to find the optimal pairs in a certain admissible set so as to minimize the compliance of the network.

As a measure of the compliance we assume the work done by the load

$$
F(\sigma)=\int_{\Omega} q w_{\sigma} d x
$$

which turns out to be a functional of $\sigma$, if $w_{\sigma}$ is denoted as the solution of (1.1) for the pretraction state $\sigma$. It is also assumed that, for practical reasons, we are interested in minimizing the compliance for a given total pretraction force $S$ applied all around the boundary, and for fiber tractions bounded away from zero and not exceeding the strength $\Lambda$ of the fibers. Thus, if $\mathscr{S}$ denotes the set of functions $\sigma(x)=\left(\sigma_{1}\left(x_{2}\right), \sigma_{2}\left(x_{1}\right)\right)$ such that

$$
\lambda \leq \sigma_{1}\left(x_{2}\right), \quad \sigma_{2}\left(x_{1}\right) \leq \Lambda,
$$

for given positive real numbers $\lambda \leq \Lambda$, and

$$
\int_{\Omega}\left(\sigma_{1}\left(x_{2}\right)+\sigma_{2}\left(x_{1}\right)\right) d x_{1} d x_{2}=S,
$$

let us consider the following optimization problem:

$$
F(\sigma)=\min _{\mathscr{f}} F \text {. }
$$

To give problem (1.5) a proper mathematical setting, in what follows we regard $\mathscr{P}$ as a subset of $L^{\infty}(\Omega)^{2}$ and its elements as generalized functions. Accordingly, and without further mention, inequalities (1.3) and all other relations involving the $\sigma$ 's in the following are required to hold almost everywhere (a.e.) in $\Omega$. It is also assumed that $\mathscr{S} \neq \varnothing$, that is, that $2 \lambda \leq S \leq 2 \Lambda$ by (1.3) and (1.4). 
In this section we deal with the existence of solutions to problem (1.5) by applying the standard argument of the Calculus of Variations.

Observe that $F$ is bounded from below since

$$
\int_{\Omega} q w_{\sigma} d x=\int_{\Omega} \sigma_{i} w_{\sigma},{ }_{i}^{2} d x \geq 0
$$

where the summation convention is assumed. Then, there are minimizing sequences and their weak* $L^{\infty}(\Omega)$ limits trivially exist and satisfy conditions (1.3) and (1.4). On the other hand, $F$ is continuous with respect to $L^{2}(\Omega)$-convergence when it is regarded as a functional of $w_{\sigma}$. By recalling that $G$-convergence of a sequence $\left\{\sigma^{k}\right\}$ to some $\sigma$ implies that the corresponding sequence of solutions $w_{\sigma^{k}}$ of problem (1.1) converges to $w_{\sigma}$ in $L^{2}(\Omega)$ (see for instance [16, 17]) it follows that $F$ is also $G$-continuous. Therefore, solutions of (1.5) exist provided that we can prove that $G$-convergence is equivalent to weak ${ }^{*}-L^{\infty}(\Omega)$ convergence on the set $\mathscr{S}$. The following theorem shows that this is true on the whole set $\Sigma \subset L^{\infty}(\Omega)^{2}$ of all pairs of functions $\sigma$ of the form $\sigma(x)=\left(\sigma_{1}\left(x_{2}\right), \sigma_{2}\left(x_{1}\right)\right)$ satisfying (1.3).

THEOREM 1.1. In $\Sigma G$-convergence topology is equivalent to weak ${ }^{*}-L^{\infty}(\Omega)$ topology.

Proof. Let $\sigma^{k}, \sigma \in \Sigma$ be such that $\sigma^{k} G$-converges to $\sigma$. Then, denoting by $H_{g}^{1}\left(\Omega^{\prime}\right)=\left\{u \in H^{1}\left(\Omega^{\prime}\right) \mid u-g \in H_{0}^{\prime}\left(\Omega^{\prime}\right)\right\}$ with $\Omega^{\prime} \subset \Omega$ and $g \in H^{1}\left(\Omega^{\prime}\right)$, one obtains from $[16,17]$

$$
\min _{H_{g}^{1}\left(\Omega^{\prime}\right)} \int_{\Omega^{\prime}} \sigma_{i}^{k} w,{ }_{i}^{2} d x \rightarrow \min _{H_{g}^{1}\left(\Omega^{\prime}\right)} \int_{\Omega^{\prime}} \sigma_{i} w,{ }_{i}^{2} d x,
$$

for every $\Omega^{\prime} \subset \Omega$ and $g \in H^{1}\left(\Omega^{\prime}\right)$. Now, observe that every $\sigma$ of the form $\left(\sigma_{1}\left(x_{2}\right), \sigma_{2}\left(x_{1}\right)\right)$ satisfies the condition

$$
\int_{\Omega^{\prime}} \sigma_{i} u,{ }_{i} \xi_{i} d x=0, \quad \forall u \in H_{0}^{1}\left(\Omega^{\prime}\right),
$$

for all $\xi \in \mathbf{R}^{2}$ and $\Omega^{\prime} \subset \Omega$. This means that the linear function $l_{\xi}(x)=\xi \cdot x$ is the stationary point, and then the minimizer of the energy functional

$$
\int_{\Omega^{\prime}} \sigma_{i} w,{ }_{i}^{2} d x
$$

on $H_{\xi}^{1}\left(\Omega^{\prime}\right)=\left\{u \in H^{1}\left(\Omega^{\prime}\right) \mid u-1_{\xi} \in H_{0}^{1}\left(\Omega^{\prime}\right)\right\}$, for every given $\xi \in \mathbf{R}^{2}$.

Talising account of this in (1.7) then yields

$$
\int_{\Omega^{\prime}} \sigma_{i}^{k} \xi_{i}^{2} d x \rightarrow \int_{\Omega^{\prime}} \sigma_{i} \xi_{i}^{2} d x, \quad \forall \Omega^{\prime} \subset \Omega, \quad \forall \xi \in \mathbf{R}^{2},
$$

that is $\sigma^{k}-\sigma$ weakly* in $L^{\infty}(\Omega)^{2}$.

Conversely, let $\left\{\sigma^{k}\right\}$ be a sequence in $\Sigma$ which converges weakly* in $L^{\infty}(\Omega)^{2}$ to some $\sigma \in \Sigma$, so that (1.9) holds. Since (1.8) holds for $\sigma^{k}$, it follows that

$$
\int_{\Omega^{\prime}} \sigma_{i}^{k} \xi_{i}^{2} d x=\min _{H_{\xi}^{1}\left(\Omega^{\prime}\right)} \int_{\Omega^{\prime}} \sigma_{i}^{k} w,{ }_{i}^{2} d x
$$


and (1.9) reads

$$
\min _{H_{\xi}^{1}\left(\Omega^{\prime}\right)} \int_{\Omega^{\prime}} \sigma_{i}^{k} w,{ }_{i}^{2} d x \rightarrow \int_{\Omega^{\prime}} \sigma_{i} \xi_{i}^{2} d x,
$$

for all $\xi \in \mathbf{R}^{2}$ and $\Omega^{\prime} \subset \Omega$. But the convergence of the minima over $H_{\xi}^{1}\left(\Omega^{\prime}\right)$ of the functionals on the left-hand side of (1.11) implies, by Theorem 3.3 of [17], the $G$-convergence of $\sigma^{k}$ to some $\sigma^{\infty}$. Therefore,

$$
\min _{H_{\xi}^{1}\left(\Omega^{\prime}\right)} \int_{\Omega^{\prime}} \sigma_{i}^{k} w,{ }_{i}^{2} d x \rightarrow \min _{H_{\xi}^{1}\left(\Omega^{\prime}\right)} \int_{\Omega^{\prime}} \sigma_{i}^{\infty} w,{ }_{i}^{2} d x,
$$

for all $\xi \in \mathbf{R}^{2}$ and $\Omega^{\prime} \subset \Omega$. By comparing (1.11) and (1.12) one obtains

$$
\min _{H_{\xi}^{1}\left(\Omega^{\prime}\right)} \int_{\Omega^{\prime}} \sigma_{i}^{\infty} w,{ }_{i}^{2} d x=\int_{\Omega^{\prime}} \sigma_{i} \xi_{i}^{2} d x .
$$

Thus, the minimum over $H_{\xi}^{1}\left(\Omega^{\prime}\right)$ of the functional corresponding to $\sigma^{\infty}$ is determined by the weak* limit $\sigma$ of $\left\{\sigma^{k}\right\}$. Then, one can apply Lemma 3.2 of [17], which states that a quadratic form with coefficients in $L^{\infty}(\Omega)$ and eigenvalues between $\lambda$ and $\Lambda$, is determined by the minima which the associated functional takes on $H_{\xi}^{1}\left(\Omega^{\prime}\right)$ for every $\Omega^{\prime} \subset \Omega$ and all the linear data $l_{\xi}$ on $\partial \Omega^{\prime}$. In the present case that theorem yields

$$
\sigma_{i}^{\infty}(x) \xi_{i}^{2}=\lim _{\rho \rightarrow 0} \frac{1}{|\Omega \rho|} \int_{\Omega_{\rho}} \sigma_{i} \xi_{i}^{2} d y, \quad \text { a.e. in } \Omega,
$$

for every $\xi \in \mathbf{R}^{2}$ and for any family $\left\{\Omega_{\rho}\right\}_{\rho>0}$ of open subsets of $\Omega$ that shrink at $x$ nicely in the sense of Rudin (cf. [22, Ch. 8], when $\rho \rightarrow 0$. A well-known property of the averages of Lebesgue functions (cf. [22, Ch. 8]), implies then that the right-hand side of $(1.14)$ is equal to $\sigma_{1}\left(x_{2}\right) \xi_{1}^{2}+\sigma_{2}\left(x_{1}\right) \xi_{2}^{2}$ a.e. in $\Omega$. So $\sigma^{\infty}=\sigma$ in $L^{\infty}(\Omega)^{2}$ and $\sigma$ is also the $G$-limit of $\left\{\sigma^{k}\right\}$.

REMARK. It is worthwhile to notice that, by the same argument, the equivalence between $G$-convergence and $L^{\infty}(\Omega)$-weak* convergence maintains for the wider class of second-order elliptic operators with coefficients $\sigma=\left[\sigma_{i j}(x)\right]$ symmetric and divergence-free in the weak sense. In fact, the energy functional is in that case

$$
\int_{\Omega} \sigma_{i j} w,{ }_{i} w,{ }_{j} d x
$$

and (1.8) becomes

$$
\int_{\Omega^{\prime}} \sigma_{i j} u,{ }_{i} \xi_{j} d x=0
$$

which is again satisfied for every $u \in H_{0}^{1}\left(\Omega^{\prime}\right)$ and for all $\xi \in \mathbf{R}^{2}$. So, optimization problems analogous to the present one can be treated in the same way. To stay with an example pertinent to tension-structures, one might consider the optimization, in the sense of the present problem, of the prestress field in a membrane transversally loaded, where the prestresses $\sigma_{i j}$ are symmetric and divergence-free by the in-plane equilibrium of the membrane. 
2. Characterization of the solutions and uniqueness of the optimal displacement. This and the next sections are devoted to the analysis of some general properties of problem (1.5). Let us use Lagrange multipliers and write Kuhn-Tucker conditions for an extremum, $[2,3,23]$. Introduce slack variables $l_{i}(z)$ and $L_{i}(z), i=1,2$, with $l_{i}, L_{i} \in L^{\infty}(0,1)$, to take the constraints (1.3) into account:

$$
\begin{gathered}
l_{i}^{2}=\sigma_{i}-\lambda \geq 0, \\
L_{i}^{2}=\Lambda-\sigma_{i} \geq 0,
\end{gathered}
$$

and the multipliers $\varphi(x)$ in $H_{0}^{1}(\Omega)^{\ddagger}, \alpha_{i}, \beta_{i}, i=1,2$, in $L^{1}(0,1)$ and $\mu \in \mathbf{R}$, respectively associated to $(1.1),(2.1)_{1},(2.1)_{2}$, and (1.4). The minima of $F$ under the constraints of the problem are then stationary points of the Lagrangian $\mathscr{L}$ :

$$
\begin{aligned}
\mathscr{L}= & \int_{\Omega} q w d x+\int_{\Omega} \varphi\left(\sigma_{i} w,_{i i}+q\right) d x+\mu\left(S-\int_{\Omega}\left(\sigma_{1}+\sigma_{2}\right) d x\right) \\
& +\int_{0}^{1}\left[\alpha_{i}(z)\left(l_{i}^{2}(z)-\sigma_{i}(z)+\lambda\right)+\beta_{i}(z)\left(L_{i}^{2}(z)+\sigma_{i}(z)-\Lambda\right)\right] d z
\end{aligned}
$$

and this requires that the following conditions are satisfied

$$
\begin{array}{ll}
\sigma_{1}\left(x_{2}\right) \varphi,{ }_{11}+\sigma_{2}\left(x_{1}\right) \varphi,_{22}=-q, & \text { in } \Omega, \\
\varphi=0, & \text { on } \partial \Omega,
\end{array}
$$

and

$$
\begin{gathered}
\int_{0}^{1} \varphi w_{\sigma},{ }_{11} d x_{1}-\mu-\alpha_{1}\left(x_{2}\right)+\beta_{1}\left(x_{2}\right)=0 \\
\int_{0}^{1} \varphi w_{\sigma},{ }_{22} d x_{2}-\mu-\alpha_{2}\left(x_{1}\right)+\beta_{2}\left(x_{1}\right)=0 .
\end{gathered}
$$

Moreover, the slack multipliers have to satisfy the complementarity conditions

$$
\begin{aligned}
& \alpha_{i}\left(\sigma_{i}-\lambda\right)=0, \\
& \beta_{i}\left(\Lambda-\sigma_{i}\right)=0,
\end{aligned}
$$

and, for a minimum of $F$, the inequalities

$$
\alpha_{i}(z) \geq 0 \text { and } \beta_{i}(z) \geq 0 .
$$

From (2.3),

$$
\varphi=w_{\sigma} .
$$

Then, when (2.7) is taken into account and (2.4) is integrated by parts, one gets

$$
\begin{aligned}
& \int_{0}^{1} w_{\sigma}^{2},{ }_{1} d x_{1}+\mu=-\alpha_{1}\left(x_{2}\right)+\beta_{1}\left(x_{2}\right), \\
& \int_{0}^{1} w_{\sigma}^{2},{ }_{2} d x_{2}+\mu=-\alpha_{2}\left(x_{1}\right)+\beta_{2}\left(x_{1}\right) .
\end{aligned}
$$

We now adapt an elegant argument of Prager and Taylor [7] (see also [4, 24]) to show that the previous conditions are sufficient for a minimum of $F$.

\footnotetext{
${ }^{\ddagger}$ Here, $\varphi$ is chosen in the dual of the space $H^{-1}(\Omega)$ where the equilibrium equation is naturally formulated. Having chosen $q$ in $L^{2}(\Omega)$ is in fact unessential till Lemma 3.1.
} 
THEOREM 2.1. If $\sigma^{*}$ and $w_{\sigma^{*}}$ satisfy conditions $(2.5)-(2.8)$ for some $\mu^{*}, \alpha_{i}^{*}$, and $\beta_{i}^{*}$, then

$$
F\left(\sigma^{*}\right) \leq F(\sigma) \quad \forall \sigma \in \mathscr{S} .
$$

Proof. Let $\sigma^{*}, w_{\sigma^{*}}$ meet Kuhn-Tucker conditions and let $\sigma, w_{\sigma}$ be any other solution of (1.1), with $\sigma \in \mathscr{S}$. Then, the following inequality holds

$$
\int_{\Omega}\left(\sigma_{i}-\sigma_{i}^{*}\right) w_{\sigma^{*},{ }_{i}}^{2} d x \leq 0 .
$$

In fact, let us consider the integral

$$
\int_{\Omega}\left(\sigma_{1}-\sigma_{1}^{*}\right) w_{\sigma^{*},{ }_{1}}^{2} d x
$$

first, and let $I_{\lambda}^{1}, I^{1}, I_{\Lambda}^{1}$ be a partition of $(0,1)$ such that

$$
\begin{array}{ll}
\sigma_{1}^{*}=\lambda, & \text { for } x_{2} \in I_{\lambda}^{1}, \\
\lambda<\sigma_{1}^{*}<\Lambda, & \text { for } x_{2} \in I^{1}, \\
\sigma_{1}^{*}=\Lambda, & \text { for } x_{2} \in I_{\Lambda}^{1} .
\end{array}
$$

It follows from the complementarity conditions and from (2.6) that

$$
\begin{array}{ll}
\alpha_{1}^{*} \geq 0 \quad \text { and } \quad \beta_{1}^{*}=0, & \text { in } I_{\lambda}^{1}, \\
\alpha_{1}^{*}=\beta_{1}^{*}=0, & \text { in } I^{1}, \\
\alpha_{1}^{*}=0 \text { and } \quad \beta_{1}^{*} \geq 0, & \text { in } I_{\Lambda}^{1} .
\end{array}
$$

By using the Fubini-Tonelli theorem in (2.11), it follows from (2.13) and (2.8) that

$$
\begin{aligned}
\int_{0}^{1}\left(\sigma_{1}-\sigma_{1}^{*}\right)\left(\int_{0}^{1} w_{\sigma^{*},}^{2}, d x_{1}\right) d x_{2} & =-\mu^{*} \int_{I^{\prime}}\left(\sigma_{1}-\sigma_{1}^{*}\right) d x_{2} \\
& +\int_{I_{\lambda}^{\prime} \cup I_{\Lambda}^{\prime}}\left(\sigma_{1}-\sigma_{1}^{*}\right)\left(\int_{0}^{1} w_{\sigma^{*},}^{2},{ }_{1} d x_{1}\right) d x_{2} .
\end{aligned}
$$

But, from $(2.8)_{1}$ and (2.13)

$$
\int_{0}^{1} w_{\sigma^{*},{ }_{1}}^{2} d x_{1} \leq-\mu^{*} \text { and }\left(\sigma_{1}-\sigma_{1}^{*}\right) \geq 0 \text { in } I_{\lambda}^{1}
$$

and

$$
\int_{0}^{1} w_{\sigma^{*},}^{2}{ }_{1} d x_{1} \geq-\mu^{*} \text { and }\left(\sigma_{1}-\sigma_{1}^{*}\right) \leq 0 \text { in } I_{\Lambda}^{1}
$$

If (2.15) is taken into account in (2.14), we have that

$$
\int_{\Omega}\left(\sigma_{1}-\sigma_{1}^{*}\right) w_{\sigma^{*},{ }_{1}}^{2} d x \leq-\mu^{*} \int_{0}^{1}\left(\sigma_{1}-\sigma_{1}^{*}\right) d x_{2} .
$$

Analogously,

$$
\int_{\Omega}\left(\sigma_{2}-\sigma_{2}^{*}\right) w_{\sigma^{*},,_{2}}^{2} d x \leq-\mu^{*} \int_{0}^{1}\left(\sigma_{2}-\sigma_{2}^{*}\right) d x_{1} .
$$

Then, inequality (2.10) follows by summing (2.16) and (2.17) if one recalls (1.4). 
Let us write (2.10) in the equivalent form

$$
\int_{\Omega} \sigma_{i}^{*} w_{\sigma^{*},}^{2}{ }_{i} d x-2 \int_{\Omega} q w_{\sigma^{*}} d x \geq \int_{\Omega} \sigma_{i} w_{\sigma^{*},}^{2}{ }_{i} d x-2 \int_{\Omega} q w_{\sigma^{*}} d x
$$

and notice that

$$
\int_{\Omega} \sigma_{i} w_{\sigma^{*},}^{2}{ }_{i} d x-2 \int_{\Omega} q w_{\sigma^{*}} d x \geq \int_{\Omega} \sigma_{i} w_{\sigma}^{2},{ }_{i} d x-2 \int_{\Omega} q \omega_{\sigma} d x
$$

because $w_{\sigma}$ is the solution of $(1.1)$ for the pair of coefficients $\sigma=\left(\sigma_{1}, \sigma_{2}\right)$. Thus,

$$
\int_{\Omega} \sigma_{i}^{*} w_{\sigma^{*},}^{2}{ }_{i} d x-2 \int_{\Omega} q w_{\sigma^{*}} d x \geq \int_{\Omega} \sigma_{i} w_{\sigma}^{2},{ }_{i} d x-2 \int_{\Omega} q w_{\sigma} d x,
$$

where, by $(1.6)_{1}$, the left-hand side and the right-hand side of (2.20) are equal to $-\int_{\Omega} q w_{\sigma^{*}} d x$ and $-\int_{\Omega} q w_{\sigma} d x$, respectively. So, one finds

$$
\int_{\Omega} q w_{\sigma^{*}} d x \leq \int_{\Omega} q w_{\sigma} d x
$$

which is $(2.9)$.

From the theorem all the stationary points of $F$ are absolute minima, so they give $F$ the same value. An important consequence of this is stated in the following theorem.

THEOREM 2.2. If $\sigma$ and $\sigma^{*}$ are two minimizers of $F$, then

$$
w_{\sigma}=w_{\sigma^{*}} .
$$

Proof. From the chain of inequalities (2.18) and (2.19), and from the equality of the first and the last term in (2.18) and (2.19), respectively, it follows that

$$
\int_{\Omega} \sigma_{i} w_{\sigma^{*}}^{2},{ }_{i} d x-2 \int_{\Omega} q w_{\sigma^{*}} d x=\int_{\Omega} \sigma_{i} w_{\sigma}^{2},_{i} d x-2 \int_{\Omega} q w_{\sigma} d x .
$$

Then, if it is observed that the total energy functional $E_{\sigma}: H_{0}^{1}(\Omega) \rightarrow \mathbf{R}$ defined by

$$
E_{\sigma}(w)=\int_{\Omega} \sigma_{i} w_{, i}^{2} d x-2 \int_{\Omega} q w d x
$$

attains an absolute minimum at $w_{\sigma},(2.23)$ implies that $w_{\sigma^{*}}$ is also a minimizer of $E_{\sigma}$. Hence, $w_{\sigma^{*}}$ coincides with $w_{\sigma}$ since the minimizer of $E_{\sigma}$ is unique.

3. Qualitative properties and uniqueness of the solution. The uniqueness of the optimal displacement $w_{\sigma}$ does not imply that the corresponding coefficients in the differential problem (1.1) are also uniquely determined. In this section we analyze some qualitative properties of problem (1.5) which are partly devoted to the discussion of uniqueness of the optimal pretractions.

Let it be assumed that the sets

$$
I_{\Lambda}^{i}=\left\{z \in(0,1) \mid \sigma_{i}(z)=\Lambda\right\}, \quad i=1,2,
$$

be intervals, for simplicity. The case where $I_{\Lambda}^{i}$ are simply measurable sets requires slight changes in the conclusions, but the argument is the same and the situation is sufficiently clear from the case treated explicitly here. 
THEOREM 3.1. If $\sigma$ is a solution to problem (1.5) and $\mu$ is the corresponding value of the multiplier in (2.8), then $\mu \geq 0$ implies

$$
q=0 \text { in } \Omega-I_{\Lambda}^{2} \times I_{\Lambda}^{1} .
$$

Moreover, $q$ is required to satisfy the conditions

$$
\int_{I_{\Lambda}^{2} \times I_{\Lambda}^{1}} q d x=\int_{I_{\Lambda}^{2} \times I_{\Lambda}^{1}} q x_{i} d x=0, \quad \text { if } \overline{I_{\Lambda}^{2} \times I_{\Lambda}^{1}} \subset \Omega,
$$

or

$$
\int_{I_{\Lambda}^{2} \times I_{\Lambda}^{1}} q x_{2} d x=0
$$

if $I_{\Lambda}^{2} \times I_{\Lambda}^{1}$ intersects $\partial \Omega$ only along the edge $x_{2}=0$. A straightforward adjustment of this condition is required if $\overline{I_{\Lambda}^{2} \times I_{\Lambda}^{1}}$ intersects $\partial \Omega$ along any other edge. No additional condition on $q$ is needed if $I_{\Lambda}^{2} \times I_{\Lambda}^{1}$ touches the boundary of $\Omega$ on more than one edge.

Proof. Let us consider $(2.8)_{1}$, first. From (2.13) we have

$$
\begin{array}{ll}
\int_{0}^{1} w_{\sigma}^{2},{ }_{1} d x_{1} \leq-\mu, & \text { for } x_{2} \in I_{\lambda}^{1}, \\
\int_{0}^{1} w_{\sigma}^{2},{ }_{1} d x_{1}=-\mu, & \text { for } x_{2} \in I^{1}, \\
\int_{0}^{1} w_{\sigma}^{2},{ }_{1} d x_{1} \geq-\mu, & \text { for } x_{2} \in I_{\Lambda}^{1} .
\end{array}
$$

If $\mu \geq 0$, then from $(3.4)_{1}, 2$ it follows that

$$
w_{\sigma, 1}=0, \quad \text { a.e. in }(0,1) \times I_{\lambda}^{1} \cup I^{1} .
$$

It is concluded, by integration and taking the boundary conditions into account, that

$$
w_{\sigma}=0 \quad \text { in }(0,1) \times I_{\lambda}^{1} \cup I^{1} .
$$

Likewise,

$$
w_{\sigma}=0 \quad \text { in } I_{\lambda}^{2} \cup I^{2} \times(0,1) .
$$

So,

$$
w_{\sigma}=0 \text { and } q=0 \text { in } \Omega-I_{\Lambda}^{2} \times I_{\Lambda}^{1},
$$

by the equilibrium equation.

Now, considering that $w_{\sigma}$ is the solution of $(1.1), w_{\sigma}$ has to minimize the total energy $E_{\sigma}($ see $(2.24))$ in $H_{0}^{1}(\Omega)$, and this requires

$$
\int_{\Omega} \sigma_{i} w_{\sigma},{ }_{i} v,{ }_{i} d x-\int_{\Omega} q v d x=0, \quad \forall v \in H_{0}^{1}(\Omega) .
$$

When recalling (3.6) and (3.7), (3.9) is written

$$
\int_{I_{\Lambda}^{2} \times I_{\Lambda}^{\prime}} \sigma_{i} w_{\sigma},{ }_{i} v,{ }_{i} d x-\int_{I_{\Lambda}^{2} \times I_{\Lambda}^{1}} q v d x=0, \quad \forall v \in H_{0}^{1}(\Omega) .
$$

Then, if $I_{\Lambda}^{2} \times I_{\Lambda}^{1}$ is internal to $\Omega$, one can choose $v$ to be any infinitesimal rigid displacement on $I_{\Lambda}^{2} \times I_{\Lambda}^{1}$ and obtain (3.3) . Analogously, one can choose $v$ so that it corresponds to a rigid rotation of $I_{\Lambda}^{2} \times I_{\Lambda}^{1}$ around the edge $x_{2}=0$ and obtain 
$(3.3)_{2}$, if this circumstance applies. No condition on $q$ is required in addition to (3.2) if neither case above applies.

Theorem 3.1 implies that $\mu$ has to be negative if $I_{\Lambda}^{2} \times I_{\Lambda}^{1}=\varnothing$, since by assumption $q$ cannot vanish in $\Omega$. Also, $\mu<0$ if $q \geq 0$ in $\Omega$ because (3.2) and (3.3), cannot be satisfied unless $q=0$. More generally, the next theorem shows that $\mu$ cannot be positive when

$$
2 \lambda<S<2 \Lambda \text {. }
$$

Notice that $\mathscr{S}$ contains just one element when $S=2 \lambda$ or $S=2 \Lambda$. So, uniqueness is trivial in these cases.

Theorem 3.2. Let $\sigma$ be optimal and let (3.11) apply. Then, $\mu \leq 0$.

Proof. Observe that (1.4) and (3.11) imply that meas $\left(I_{\lambda}^{i} \cup I^{i}\right)>0$ for at least one value of $i$. Let

$$
\operatorname{meas}\left(I_{\lambda}^{1} \cup I^{1}\right)>0,
$$

so that at least one out of $I_{\lambda}^{1}$ and $I^{1}$ is nonempty. Then, the statement follows from $(3.4)_{1}$ or $(3.4)_{2}$.

The situation in Theorem 3.1 suggests cases where there is nonuniqueness.

TheOREM 3.3 (Nonuniqueness). Let $\sigma$ be optimal with $\mu=0$ and let (3.11) apply. Then, there are pretractions $\sigma^{*} \neq \sigma$ which are still optimal.

Proof. Condition (3.2) in Theorem 3.1 and the assumption $\|q\| \neq 0$ imply that $I_{\Lambda}^{2} \times I_{\Lambda}^{1} \neq \varnothing$. Moreover, meas $\left(\Omega-I_{\Lambda}^{2} \times I_{\Lambda}^{1}\right)>0$ because $S<2 \Lambda$. Then, it is obviously possible to modify $\sigma$ so that the new pretraction state $\sigma^{*}$ is different from $\sigma$ on a subset of positive measure in $\Omega-I_{\Lambda}^{2} \times I_{\Lambda}^{1}$ and satisfies the conditions

$$
\begin{gathered}
\sigma_{1}^{*}=\sigma_{2}^{*}=\Lambda, \quad \text { in } I_{\Lambda}^{2} \times I_{\Lambda}^{1}, \\
\lambda \leq \sigma_{1}^{*}, \sigma_{2}^{*} \leq \Lambda \quad \text { in } \Omega-I_{\Lambda}^{2} \times I_{\Lambda}^{1}, \\
\int_{\Omega}\left(\sigma_{1}^{*}+\sigma_{2}^{*}\right) d x=S .
\end{gathered}
$$

So, $\sigma^{*} \in \mathscr{S}$. Moreover, $w_{\sigma}$ is also the solution of problem (1.1) under the pretractions $\sigma^{*}$, since the displacement $w_{\sigma}$ vanishes in $\Omega-I_{\Lambda}^{2} \times I_{\Lambda}^{1}$, where $\sigma$ may be different from $\sigma^{*}$, whereas it is different from 0 only where $\sigma=\sigma^{*}$. Thus, $\sigma^{*}$ also is a minimizer of $F$ under the same data $q$ and $\mathscr{S}$.

It is not difficult to see that, for given values of $\lambda$ and $\Lambda$, one can construct problems (1.5) with nonunique solution by suitably adjusting $q$ and $S$. In fact, let us fix a rectangle $R=(a, b) \times(c, d) \subset \subset \Omega$ and choose $w_{\Lambda} \in H^{2}(\Omega)$ such that $w_{\Lambda}=0$ in $\Omega-R$. Then let $q$ be defined by

$$
-q_{\Lambda}=\Lambda\left(w_{\Lambda},{ }_{11}+w_{\Lambda},{ }_{22}\right)
$$

in $\Omega$. It follows that $w_{\Lambda}$ is the deflection of the net under the load $q_{\Lambda}$ for pretractions $\sigma_{\Lambda}$ arbitrarily chosen in $\Omega-R$, and such that

$$
\sigma_{\Lambda 1}=\sigma_{\Lambda 2}=\Lambda \text { in } R \text {. }
$$


In particular, since $w_{\Lambda}$ vanishes in $\Omega-R$, the energy equality is written as

$$
\int_{\Omega} q_{\Lambda} w_{\Lambda} d x=\int_{\Omega} \Lambda\left|\nabla w_{\Lambda}\right|^{2} d x
$$

Now, if $\tilde{\sigma}$ is any other distribution of pretractions equipollent to $\sigma_{\Lambda}$ with $\tilde{\sigma}_{i} \leq \Lambda$ in $\Omega$, and if $w_{\tilde{\sigma}}$ is the corresponding deflection of the net under the load $q_{\Lambda}$, one obtains

and also

$$
\int_{\Omega} q_{\Lambda} w_{\tilde{\sigma}} d x=\int_{\Omega} \tilde{\sigma}_{i} w_{\tilde{\sigma}}^{2},{ }_{i} d x
$$

$$
\int_{\Omega} \Lambda\left|\nabla w_{\Lambda}\right|^{2} d x \leq \int_{\Omega} \tilde{\sigma}_{i} w_{\tilde{\sigma}}^{2},{ }_{i}^{\prime} d x
$$

from the monotonicity of the stored energy with respect to $\sigma$ (see, e.g., [14]). It follows that

$$
\int_{\Omega} q_{\Lambda} w_{\Lambda} d x \leq \int_{\Omega} q_{\Lambda} w_{\tilde{\sigma}} d x
$$

which shows that $\sigma_{\Lambda}$ is in fact optimal for the given load $q_{\Lambda}$. But $\sigma_{\Lambda}$ can be arbitrarily chosen in $\Omega-R$, if $S>[(b-a)+(d-c)]+\lambda[2-(b-a)-(d-c)]$; so one has a problem for which the optimal pretractions are not unique.

We remark that the lack of uniqueness shows that $F(\sigma)$ is not strictly convex in general.

Let us discuss now the case $\mu<0$ in Theorem 3.2. Our aim is to describe features of the optimal pretraction fields in this instance. The following lemma is needed:

LEMmA 3.1. For every $w \in H^{2}(\Omega) \cap H_{0}^{1}(\Omega)$,

$$
\begin{aligned}
\int_{0}^{1} w_{, 1}^{2}\left(x_{1}, z\right) d x_{1} & =2 \int_{0}^{1}\left(\int_{0}^{z} w,{ }_{1} w,_{12} d x_{2}\right) d x_{1} \\
& =2 \int_{0}^{1}\left(\int_{0}^{z} w,{ }_{1} w,{ }_{12} d x_{2}\right) d x_{1} .
\end{aligned}
$$

Proof. Equalities (3.12) trivially hold for a function $w \in C^{2}(\bar{\Omega})$ that vanishes on $\partial \Omega$. Let $\Omega_{z}=(0,1) \times\{z\}$ for $z \in(0,1)$. Then, by the Sobolev imbedding theorem,

$$
H^{2}(\Omega) \rightarrow H^{1, p}\left(\Omega_{z}\right), \quad 2 \leq p<\infty,
$$

and the imbedding is continuous. It follows that the first term in (3.12) is continuous in the topology of $H^{2}(\Omega)$; on the other hand, the remaining terms in (3.12) are also continuous in the same topology. Therefore, the equality maintains in $H^{2}(\Omega) \cap H_{0}^{1}(\Omega)$ by density.

The coefficients $\sigma_{i}$ and the domain $\Omega$ satisfy the conditions of Theorem 17.6 of Ladyzhenskaya and Ural'tseva [25]. In addition, the uniqueness theorem holds in $H_{0}^{1}(\Omega)$. It follows that $w \in H^{2}(\Omega) \cap H_{0}^{1}(\Omega)$ for every $q \in L^{2}(\Omega)$. Thus, (3.12) applies to $w$ and the integrals on the right-hand side are continuous in $z$ since $w_{\sigma},{ }_{1} w_{\sigma},{ }_{12} \in L^{1}(\Omega)$. It follows that

$$
\int_{0}^{1} w_{\sigma, 1}^{2}\left(x_{1}, z\right) d x_{1}
$$


also belongs to $C^{0}(0,1)$ when it is regarded as a function of $z$. Furthermore, from (3.12)

$$
\left.\lim _{\substack{z \rightarrow 0 \\ z \rightarrow 1}} \int_{0}^{1} w_{\sigma}, x_{1}, z\right) d x_{1}=0 \text {. }
$$

The same argument applies to $\int_{0}^{1} w_{\sigma, 2}^{2}\left(z, x_{2}\right) d x_{2}$. We are thus in a position to prove

Theorem 3.4. Let $\sigma$ be optimal, with $\mu<0$. Then the sets $I_{\lambda}^{i}, i=1,2$ have positive measure.

Proof. Let us prove that meas $I_{\lambda}^{1}>0$. Observe that by $(2.8)_{1},(2.5)$, and (2.6)

$$
\int_{0}^{1} w_{\sigma, 1}^{2}\left(x_{1}, z\right) d x_{1}+\mu<0 \leftrightarrow \alpha_{1}(z)>0 .
$$

Since from the complementarity conditions $\sigma_{1}(z)=\lambda$ when $\alpha_{1}(z)>0$; it follows that

$$
A_{\lambda}=\left\{z \in(0,1) \mid \int_{0}^{1} w_{\sigma, 1}^{2} d x_{1}+\mu<0\right\} \subset I_{\lambda}^{1} .
$$

But $A_{\lambda}$ is nonempty by (3.13) and by the assumption that $\mu<0$; moreover $A_{\lambda}$ is open because the function $\int_{0}^{1} w_{\sigma}^{2},{ }_{1} d x_{1}$ is continuous. Therefore, $I_{\lambda}^{1}$ has a positive measure.

Notice that from the same argument $A_{\lambda}$ contains left and right neighborhoods of the endpoints of the interval $(0,1)$. So, one also obtains

TheOREM 3.5. Under the hypothesis of Theorem 3.4 , there is a $\delta>0$ such that

$$
\sigma_{1}(z)=\sigma_{2}(z)=\lambda \text { for } z \in(0, \delta) \cup(1-\delta, 1) \text {. }
$$

Thus, the optimal pretractions are to be as small as possible in a strip of positive width all around the boundary of $\Omega$. Since at the boundary the deformability is limited because of the nearness of the support, the result fits with physical intuition, although the fact that the strip has a positive width does not seem obvious to us. It has not been checked whether this feature of the optimal pretraction state is familiar to people working in structure theory. It would be interesting to estimate the extension of that zone in terms of the other data $q, S$, and $\Lambda$.

Apart from practical implications, the property established in (3.16) ( an be useful to treat uniqueness when $\mu<0$, or to characterize cases where uniqueness holds. As a contribution to the understanding of this point, one should conclude with a few remarks that illustrate how (3.16) can be used in dealing with it.

It can be shown that $\mu$ and hence $\alpha_{i}$ and $\beta_{i}$ are unique when $\mu<0$. Let then $\sigma$ and $\sigma^{*}$ be minimizers of $F$ corresponding to a negative multiplier $\mu$. Since the optimal displacement in both cases is the same, call it $w$, we have from the equilibrium equation

$$
\sigma_{1} w,_{11}+\sigma_{2} w,_{22}=\sigma_{1}^{*} w,_{11}+\sigma_{2}^{*} w,_{22} \text {. }
$$

If one concentrates, for instance, on the strip $(0,1) \times(1-\delta, 1)$ where $\sigma_{1}=\sigma_{1}^{*}=\lambda$ by Theorem 3.5 , it follows that

$$
\sigma_{2}^{*}\left(x_{1}\right) w,_{22}\left(x_{1}, z\right)=\sigma_{2}\left(x_{1}\right) w,_{22}\left(x_{1}, z\right), \quad \text { for } z \in(1-\delta, 1) .
$$


Then, $\sigma_{2}^{*} \neq \sigma_{2}$ implies $w,{ }_{22}=0$. So, nonunique tractions may occur only in fibers that remain straight in a strip of width $\delta$ all around $\partial \Omega$. A fact that looks rather exceptional and suggests that uniqueness is likely to hold when $\mu<0$. Conditions on $q$ which are sufficient for uniqueness can be easily worked out and seem to cover fairly general situations, but we are unable to put them on a systematic basis. The following example points out the sort of difficulties that may arise if one allows for nonuniqueness when $\mu<0$. Let $\sigma$ and $\sigma^{*}$ be two distinct solutions, for $q=$ const, and let the set $\left\{x_{1} \in(0,1) \mid \sigma_{2}^{*}\left(x_{1}\right) \neq \sigma_{2}\left(x_{1}\right)\right\}$ contain an interval $V$. Then, $w,{ }_{22}=0$ in $V \times(0, \delta) \cup(1-\delta, 1)$. So, the integration with respect to $x_{1}$ of the equation

$$
\sigma_{1}\left(x_{2}\right) w,_{11}\left(x_{1}, x_{2}\right)=-q
$$

in $V \times(1-\delta, 1)$, yields

$$
w=-q x_{1}^{2} / 2 \sigma_{1}\left(x_{2}\right)+f_{1}\left(x_{2}\right) x_{1}+f_{2}\left(x_{2}\right),
$$

where $f_{1}$ and $f_{2}$ are arbitrary functions of $x_{2}$. It follows that $w$ may not vanish identically for $x_{2} \rightarrow 1$, as required by the boundary conditions.

Acknowledgment. This work has been partially supported by a grant of the Italian Ministry of Education to the University of Udine. The authors would like to thank also the Italian Foreign Ministry for funding the participation of C.-Q. Ru in a doctoral program organized at the International Centre for Mechanical Sciences (C.I.S.M.) during the academic year 1986/87.

\section{REFERENCES}

[1] R. Kohn and G. Strang, Optimal design and relaxation of variational problems, Comm. Pure Appl. Math. 34, Part I 113-137, Part II 139-182, Part III 353-377 (1986)

[2] N. V. Banichuk, Problems and Methods of Optimal Structural Design, Plenum Press, New York, 1983

[3] N. Olhoff and J. E. Taylor, On structural optimization, Trans. ASME Ser. E. J. Appl. Mech. 50, 1139-1151 (1983)

[4] N. C. Huang, Optimal design of elastic structures for maximum stiffness, Internat. J. Solids and Structures 17, 305-311 (1981)

[5] K. T. Cheng and N. Olhoff, An investigation concerning optimal design of solid elastic plates, Internat. J. Solids and Structures 17, 305-323 (1981)

[6] N. C. Huang, Optimal design of elastic beams for minimum-maximum deflection, J. Appl. Mech. 38, 1078-1081 (1971)

[7] W. Prager and J. E. Taylor, Problems of optimal structural design, J. Appl. Mech. 35, 102-106 (1968)

[8] R. Reiss, Optimal compliance criterion for axisymmetric solid plates, Internat. J. Solids and Structures 12, 319-329 (1976)

[9] K. A. Lurie and A. V. Cherkaev, G-closure of some particular sets of admissible material characteristics for the problem of bending of thin elastic plates, J. Optim. Theory Appl. 42, 305-316 (1984)

[10] K. A. Lurie, A. V. Cherkaev, and A. V. Fedorov, Regularization of optimal design problems for bars and plates, J. Optim. Theory Appl. 37. Part 1 499-522, Part 2 523-543 (1982)

[11] K. A. Lurie, A. V. Cherkaev, and A. V. Fedorov, On the existence of solutions to some problems of optimal design for bars and plates, J. Optim. Theory Appl. 42, 247-282 (1984)

[12] K. A. Lurie and A. V. Cherkaev, Optimal structural design and relaxed controls, Optimal Control Appl. Methods 4, 387-392 (1983) 
[13] F. Murat and L. Tartar, Calcul des variations et homogénéization, Cours de l'Ecole d'Eté d'Analyse Numérique CEA-EDF-INRIA sur l'homogénéisation, Bréau sans Nappe, Juillet 1983, Eyrolles, Paris, 1984

[14] E. Cabib and G. Dal Maso, On a class of optimum problems in structural design, J. Optimization Theory Appl. 56, 39-65 (1988)

[15] R. S. Rivlin, Plane strain of a net formed by inextensible cords, J. Rational Mech. Anal. 4, 951-974 (1955)

[16] S. Spagnolo, Convergence in energy for elliptic operators, Proc. 3rd Symp. Numer. Solut. Partial Diff. Equat., College Park, Md., 1975, ed. by B. Hubbard, Academic Press, New York, 1976, pp. 469-498

[17] E. De Giorgi and S. Spagnolo, Sulla convergenza degli integrali dell'energia per operatori ellittici del secondo ordine, Boll. U.M.I. (4) 8, 391-411 (1973)

[18] W. Velte and P. Villaggio, Are the optimum problems in structural design well posed?, Arch. Rational Mech. Anal. 78, 199-211 (1982)

[19] F. Murat, Un contre-exemple pour le problème du contrôle dans les coefficients, C. R. Acad. Sci. Paris, Ser. A 273, 708-711 (1971)

[20] F. Murat, Contre-exemples pour divers problèmes où le contrôle intervient dans les coefficients, Ann. Mat. Pura Appl. (4) 112, 49-68 (1977)

[21] I. N. Rozvany, N. Olhoff, K. T. Cheng, and J. E. Taylor, On the solid plate paradox in structural optimization, J. Struct. Mech. 10, 1-32 (1982)

[22] W. Rudin, Real and complex analysis, McGraw Hill, New York, 1966

[23] H. W. Kuhn and A. W. Tucker, Nonlinear programming, Proc. of the 2nd Berkeley Symp. on Math. Statistic and Probability, J. Wiley, Berkeley, Univ. of California, 1951

[24] J. E. Taylor, Maximum strength elastic structural design, Journal of the Engineering Mechanics Division, ASCE, 95, No. EM3, 653-663 (1969)

[25] O. A. Ladizheskaya and N. N. Ural'tseva, Linear and Quasilinear Elliptic Equations, Academic Press, New York, 1968 